\title{
Uso do corante azul de metileno a $2 \%$ na localização do linfonodo axilar em cadelas (Canis familiaris - Linnaeus, 1758)
}

\author{
Use of $2 \%$ methylene blue dye for location of axillary lymph node in bitches (Canis familiaris \\ - Linnaeus, 1758)
}

\author{
Tábata MAUÉS ${ }^{1}$; Carolina Bistritschan ISRAEL ${ }^{2}$; Maria de Lourdes Gonçalves FERREIRA ${ }^{3}$; Ana Maria Reis \\ FERREIRA $^{3}$
}

\author{
${ }^{1}$ Universidade Federal Fluminense, Faculdade de Veterinária, Niterói - RJ, Brasil \\ ${ }^{2}$ Médica veterinária autônoma, Rio de Janeiro - RJ, Brasil \\ ${ }^{3}$ Universidade Federal Fluminense, Faculdade de Veterinária, Departamento de Patologia e Clínica Veterinária, Niterói - RJ, Brasil
}

\begin{abstract}
Resumo
A biopsia de linfonodo sentinela (LS) é cada vez mais utilizada para a determinação do estadiamento clínico tumoral. O acometimento de linfonodos em animais com doença oncológica possui forte valor prognóstico no tumor da glândula mamária. Em medicina veterinária faltam diretrizes bem estabelecidas para a avaliação do LS. O objetivo deste estudo foi avaliar a eficácia do corante azul de metileno a $2 \%$ na identificação do linfonodo axilar em cadelas com lesões mamárias. Para tanto, foram utilizadas 101 cadelas, portadoras de lesões mamárias, submetidas à mastectomia radical unilateral ou bilateral. Antes do início da cirurgia, aplicou-se, por via intradérmica, na área ao redor da mama torácica cranial, solução de azul de metileno a $2 \%$ estéril. O corante foi eficaz na identificação do linfonodo axilar em 76,27\% das cadeias mamárias. Obtiveram-se estruturas coradas em azul, semelhantes a linfonodos em 78,81\% dos casos. Dessas estruturas, 96,77\% foram confirmadas ao exame histopatológico como linfonodos. Dada a importância prognóstica do comprometimento de linfonodos em alguns tumores, a avaliação do LS deve ser incorporada à rotina clínica, de modo a permitir um estadiamento mais preciso das cadelas com neoplasia mamária.
\end{abstract}

Palavras-chave: Sentinela. Neoplasia. Metástase. Mama.

\begin{abstract}
A biopsy of sentinel lymph node (SLN) is increasingly used to determine tumor clinical staging. The involvement of lymph nodes in veterinary patients has strong prognostic value in mammary gland tumors. There is a lack of well established guidelines for the evaluation of the SLN in veterinary medicine. The purpose of this research was to evaluate the efficacy of $2 \%$ methylene blue dye in identifying axillary lymph node in dogs. Thus, we used 101 bitches suffering from breast lesions that underwent unilateral or bilateral mastectomy. Before surgery, sterile $2 \%$ methylene blue solution was applied intradermally in the area around the cranial thoracic gland. The dye was effective in identifying axillary lymph node in $76.27 \%$ of mammary chains. Blue stained structures similar to lymph nodes were obtained in $78.81 \%$ of cases and $96.77 \%$ of these structures were confirmed histopathologically as lymph nodes. Given the prognostic significance of lymph node involvement in some tumors, evaluation of SLN should be incorporated into clinical routine in order to allow a more accurate clinical staging of bitches with breast cancer.
\end{abstract}

Keywords: Sentinel. Cancer. Metastasis. Breast.

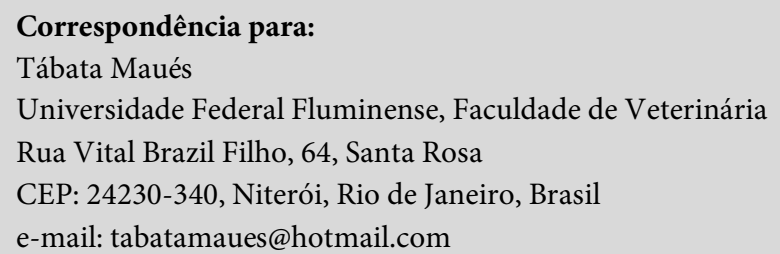

Recebido: 07/01/2015

Aprovado: 06/02/2016 


\section{Introdução}

O estudo da drenagem linfática no planejamento terapêutico do câncer de mama em humanos foi descrito por Henry François Lê Dran, que em meados do século XVIII descreveu a progressão da doença pela via linfática. No século XIX, aplicando esses conhecimentos, Halsted desenvolveu a mastectomia com ressecção dos músculos peitorais e o esvaziamento dos linfonodos axilares, denominada ressecção em monobloco (PINHEIRO et al., 2003). O conceito de mapeamento linfático e biopsia do linfonodo sentinela (LS) foi descrito pela primeira vez em humanos em casos de câncer peniano, nos quais verificou-se que o estado do LS era preditivo do tempo de sobrevida (TUOHY et al., 2009).

O LS é o primeiro linfonodo dentro de uma rede linfática que drena um tumor primário. $\mathrm{O}$ estado da doença do LS é importante para a predição da sobrevida. A identificação e a biopsia do LS são fundamentais para o estadiamento de cânceres humanos e ajuda a determinar os planos de tratamento e o prognóstico (TUOHY et al., 2009).

A drenagem linfática das mamas em cadelas foi descrita inicialmente por Baum (1918 apud PATSIKAS et al., 2006, p. 233), que corou os vasos linfáticos com nanquim aplicado ao redor da papila mamária. A drenagem linfática das cadeias mamárias é complexa (LANA et al., 2007) e embora possa ocorrer entre todas as glândulas, estudos indicam a existência de maior quantidade de conexões entre as glândulas proximais (torácicas cranial e caudal) e entre as caudais (abdominal caudal e inguinal) (SAUTET et al., 1992; PEREIRA et al., 2003). As glândulas mamárias torácicas, abdominal cranial e, ocasionalmente, a abdominal caudal drenam para os linfonodos axilares e esternais craniais (PEREIRA et al., 2003; LANA et al., 2007).

Os linfonodos axilares estão localizados no tecido adiposo da face medial da porção distal do músculo redondo maior, na região da primeira e da segunda costelas. Estão situados ventralmente à artéria e à veia toracodorsal. O linfonodo axilar raramente é avaliado, a não ser quando palpável devido aos riscos associados à extirpação do plexo braquial e da artéria e veia axilares (TUOHY et al., 2009). O tamanho desses linfonodos em cadelas varia de 0,5 a $5,0 \mathrm{~cm}$ (PINHEIRO et al., 2003) e um segundo linfonodo pode estar presente. Além das glândulas mamárias torácicas, o linfonodo axilar também drena pele, subcutâneo e músculo cutâneo do tronco, assim como pele, subcutâneo e parte da musculatura do membro torácico (JOHNSTON, 2007).

A biopsia de linfonodo sentinela é cada vez mais utilizada para a determinação do estadiamento clínico e detecção de micrometástases ocultas. Micrometástase refere-se à disseminação sistêmica de células tumorais, formando focos microscópicos e, consequentemente, indetectáveis por exames de imagem radiográfico, ultrassonográfico ou tomográfico. Acredita-se que as micrometástases sejam responsáveis por doença metastática em pacientes inicialmente diagnosticados com linfonodo sem comprometimento metastático (TUOHY et al., 2009).

Em medicina veterinária faltam diretrizes bem estabelecidas para a avaliação do LS. O acometimento de linfonodos em animais com doença oncológica possui forte valor prognóstico no tumor da glândula mamária influenciando na sobrevida global e no intervalo livre de doença (YAMAGAMI et al., 1996; BARBOSA et al., 2010; SZCZUBIAŁ; ŁOPUSZYNSKI, 2011 ).

Em humanos têm sido destacados os benefícios da utilização de várias técnicas de identificação e biópsia do LS no melanoma e no câncer de mama. Assim, já há uma clara indicação para a adaptação dessas técnicas para o uso em animais com câncer, para os quais a prática da avaliação do LS é de grande relevância clínica (TUOHY et al., 2009). Em cães as técnicas de identificação linfática descritas incluem: uso de hemossiderina (PINHEIRO et al., 2009; BESERRA et al., 2013); linfocintilografia com dextrano ${ }^{9 \mathrm{~mm} T c}$ (PEREIRA et al., 2008); linfografia com o contraste lipossolúvel Lipidol (Guerbet, 
France) (PATSIKAS et al., 2006); azul patente V e Tecnécio ${ }^{99 \mathrm{~m}} \mathrm{Tc}$ (PINHEIRO et al., 2003; BESERRA et al., 2011); solução de tinta alcoólica (PEREIRA et al., 2003); fluoresceína sódica $5 \%$ e luz ultravioleta (RAHAL et al., 1995).

O presente trabalho foi delineado para avaliar a eficácia do emprego do corante azul de metileno a $2 \%$ para a identificação do linfonodo axilar em cadelas com lesões mamárias.

\section{Material e Métodos}

O projeto foi encaminhado para avaliação pelo Comitê de Ética no Uso de Animais da Universidade Federal Fluminense (CEUA / UFF) e obteve aprovação sob o protocolo n ${ }^{\circ}$ 198/2012.

Foram utilizadas 101 cadelas adultas, de raças variadas, incluindo animais sem raça definida (SRD), portadoras de lesões mamárias, submetidas à mastectomia uni ou bilateral (HEDLUND, 2007; WHITE, 2007). Os animais foram selecionados na ocasião do atendimento oncológico no Hospital Universitário de Medicina Veterinária Professor Firmino Mársico Filho (HUVET-UFF), no período de janeiro 2011 a outubro de 2012. A coleta de amostra da lesão mamária foi efetuada por punção por agulha fina (PAF), preparo e coloração das lâminas pela técnica de May Grünwald-Giemsa (Alemanha) e posterior avaliação citopatológica. Foram incluídos no estudo animais com diagnóstico citopatológico sugestivo de neoplasia benigna ou maligna. As cadelas foram submetidas aos exames pré-operatórios de rotina: exame radiográgico de tórax em três posições (lateral direita, lateral esquerda e ventro-dorsal) e exame ultrassonográfico abdominal para pesquisa de metástases durante o pré-operatório. Além disso, também foi realizada a avaliação hematológica e a cardiológica com eletrocardiografia e, conforme a necessidade o ecodopplercardiograma, para avaliar o risco anestésico-cirúrgico.

Os animais incluídos no trabalho apresentavam as seguintes características: portadores de nódulos mamários com ausência de metástases visíveis aos exames de imagem; ausência de alterações nos demais exames que impossibilitassem o procedimento anestésico-cirúrgico; e com linfonodos axilares sem alterações aparentes ao exame físico. Os que apresentaram suspeita de carcinoma inflamatório à primeira avaliação clínica foram excluídos do estudo em função da contraindicação cirúrgica por conta do intenso envolvimento cutâneo e da coagulopatia associados a essa neoplasia.

Foram selecionadas cadelas nos estágios I, II, III e IV de estadiamento clínico neoplásico $\left(\mathrm{OWEN}^{1}\right.$, 1980), ou seja, pacientes sem evidências de metástase distante aos exames de imagem.

A solução estéril de azul de metileno a $2 \%$ foi aplicada, pela via intradérmica, cerca de 5 minutos antes do início do procedimento cirúrgico (CAMPOS et al., 2007). A seringa utilizada foi a de $1,0 \mathrm{~mL}$ com agulha $13 \times 4,5 \mathrm{~mm}$. Foram administrados $0,5 \mathrm{~mL}$ de azul de metileno para cadelas com até $15 \mathrm{~kg}$ e $1,0 \mathrm{~mL}$ para cadelas com peso superior a $15 \mathrm{~kg}$, em duas ou mais aplicações para a distribuição do volume do corante que foi aplicado na região periareolar $\mathrm{da}(\mathrm{s})$ mama(s) torácica(s) cranial(is) da cadeia mamária acometida pela tumoração.

A biópsia excisional do linfonodo axilar ipsilateral à(s) mama(s) afetada(s) foi realizada no transoperatório, após a retirada da cadeia mamária, variando de 30 a 120 minutos após a aplicação do corante. A partir da incisão da mastectomia foi realizada a divulsão cuidadosa do tecido subcutâneo e adiposo da região axilar. Quando localizado, o linfonodo era tracionado (Figura 1) após divulsão do tecido subcutâneo circunjacente; quando necessário foi aplicada a ligadura ao pedículo vascular e aos vasos linfáticos eferentes, utilizando-se um fio absorvível poliglactin 910 caudalmente ao ponto de resseção; os vasos eram resseccionados, liberando o nodo linfático que era removido por completo.

\footnotetext{
Sitema estadiamento clínico modificado por Lana, Rutterman and Withrow(2007) a partir de estudo da Organização Mundial de Saúde (OWEN, L. N. Classification of tumors in domestic animals. Geneva: World Health Organization, 1980).
}

Braz. J. Vet. Res. Anim. Sci., São Paulo, v. 53, n. 1, p. 32-38, 2016 


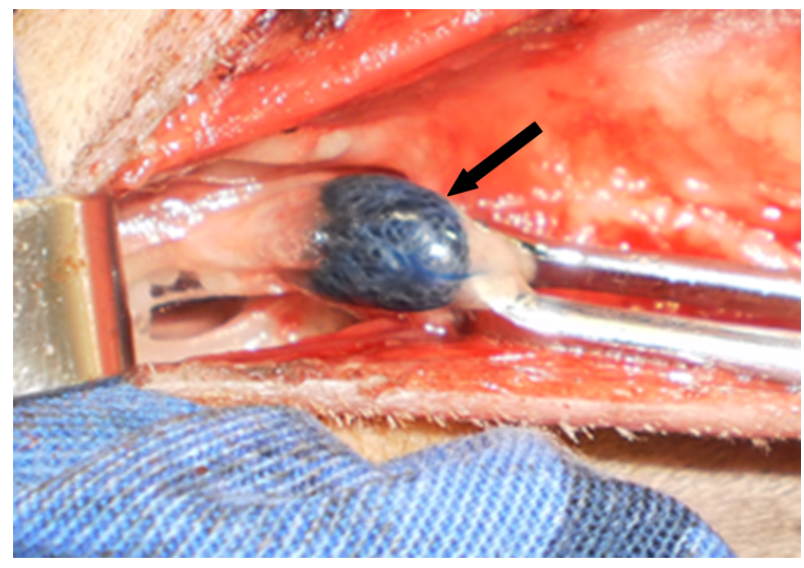

Figura 1 - Linfonodo axilar direito (seta) de cadela corado por azul de metileno a $2 \%$

Nos animais portadores de linfonodos axilares acessórios a remoção de tais linfonodos foi realizada conforme descrito anteriormente. Os linfonodos axilares coletados foram fixados em solução aquosa de formol tamponado a $10 \%$, clivados, processados e incluídos em parafina histológica. Cortes de $5 \mu \mathrm{m}$ de espessura foram confeccionados em micrótomo ${ }^{2}$ rotativo e corados por hematoxilina-eosina (HE) para avaliação em microscópio de luz ${ }^{3}$. A análise histopatológica avaliou as amostras como tecido linfático proveniente de linfonodo ou tecido não linfático.

\section{Resultados e Discussão}

O corante azul de metileno a $2 \%$ foi aplicado em 101 cadelas e 118 cadeias mamárias, visto que 17 animais foram submetidos a mastectomia bilateral no mesmo tempo cirúrgico. Durante o procedimento cirúrgico, a partir das cadeias mamárias, foram obtidas estruturas coradas em azul semelhantes a linfonodos em 78,81\% (93/118), das quais 96,77\% (90/93) foram confirmadas histopatologicamente como linfonodos e 3,23\% (3/93) como tecido não linfóide (tecido adiposo ou musculatura). Em 21,19\% dos casos (25/118) não foram identificadas estruturas

\footnotetext{
2 Micrótomo Spencer, marca América Optical (Southbridge, Massachusetts, EUA)

${ }^{3}$ Olympus BX41, marca Olympus (Southend-on-Sea, Reino Unido).
}

coradas em azul durante a cirurgia. Desta forma, o corante foi eficaz na identificação do linfonodo axilar em 76,27\% (90/118) dos casos. Já Pinheiro et al. (2003) utilizando marcação com azul patente associado ao Tecnécio ${ }^{99 \mathrm{~m}} \mathrm{Tc}$ em caninos localizaram o linfonodo sentinela em 94,5\% (35/37) dos casos e utilizando apenas o azul patente localizaram 71,43\% (5/7) dos casos, aproximando-se mais dos resultados obtidos neste estudo. As vantagens do emprego de radiofármacos como o Tecnécio ${ }^{99 \mathrm{~m}} \mathrm{Tc}$ são a obtenção de uma maior sensibilidade em relação ao uso de corantes isolados e a identificação do LS em locais não previstos, porém são fármacos que necessitam de equipe e equipamentos especializados e com alto custo quando comparados ao uso dos corantes isolados como o azul de metileno.

Em 23,73\% (28/118) dos casos, não foi localizado o linfonodo axilar corado. Este fato pode ser consequência do tempo consumido entre a aplicação do corante e a procura pelo linfonodo; da ausência de marcação do corante; ou da dificuldade que o cirurgião encontrou para identificar o linfonodo em localização não previsível. A detecção do linfonodo sentinela em localizações atípicas pode ser difícil (SAPIENZA et al., 2004).

Nickel et al. (1981) referem que o linfonodo axilar acessório está presente em $25 \%$ dos animais, localizando-se junto à borda dorsal do músculo peitoral profundo ou ventral do longuíssimo dorsal. Porém, no presente trabalho o linfonodo acessório foi detectado em apenas 4,45\% (4/90) dos casos. Essa diferença em relação aos dados da literatura pode estar relacionada ao fato de parte dos animais estudados não possuírem o linfonodo acessório; ao linfonodo estar em localização atípica; ou ao linfonodo não ter sido corado.

Corantes como o azul de metileno e o verde brilhante são evitados em medicina humana por apresentar rápida difusão após injeção e estarem associados ao aparecimento de mastite por necrose gordurosa (QUADROS; GEBRIM, 2007). No presente trabalho, as cadelas foram submetidas à mastectomia 
com remoção de todo o tecido mamário ipsilateral ao linfonodo coletado, o que reduz os riscos de mastite após a aplicação do corante azul de metileno. Tais complicações não foram observadas no pósoperatório dos animais. As únicas alterações identificadas foram reações alérgicas na pele do ponto de aplicação do corante em dois animais (1,98\% dos animais que receberam o corante). Reações alérgicas também foram relatadas em humanos com uso do corante azul patente (SLETH, 2008). Reações em caninos não foram observadas com uso de outros marcadores como azul patente e hemossiderina (BESERRA et al., 2013).

A pele de humanos e caninos apresenta uma rica rede de vasos linfáticos, localizados principalmente na camada mais superficial da derme, próximo à epiderme. A injeção subdérmica ou intradérmica de diferentes substâncias permite o estudo da drenagem linfática, com algumas variações de velocidade de drenagem e da retenção linfática, dependendo das características físicas do composto injetado. A progressão nas vias linfáticas depende principalmente do tamanho das partículas, com progressão daquelas de maior peso molecular em menor quantidade e de forma mais lenta (SAPIENZA et al., 2004). Desta forma, optou-se pela administração do corante pela via intradérmica. A aplicação do azul de metileno foi realizada na derme sobrejacente à mama torácica cranial, na região periareolar. Quando o animal, por particularidade anatômica, não possuía a referida mama, a aplicação foi efetuada na única mama torácica presente. Sabe-se que o intervalo necessário para a visualização do linfonodo corado é variável (SAPIENZA et al., 2004), porém em todos os casos analisados houve a difusão do corante na derme adjacente imediatamente após a aplicação. O tempo entre a aplicação do corante e a biópsia do linfonodo variou em função do tempo de cirurgia situado entre 30 minutos a cerca de duas horas. A biópsia foi iniciada após a remoção completa do tecido mamário com a mastectomia e antes do início da aproximação do tecido subcutâneo para oclusão da ferida, diferente da conduta adotada por outros autores que efetuaram a linfonodectomia logo após a aplicação do corante e antes do início da mastectomia (KHATIB et al., 2011).

Em todos os animais foi confirmada a difusão do corante em derme e tecido subcutâneo, incluindo os animais em que o linfonodo axilar corado não foi visualizado.

Conclui-se que o corante azul de metileno a $2 \%$ administrado pela via intradérmica na região periaureolar da mama torácica cranial difundiu-se pela cadeia linfática, sendo eficaz para a identificação do linfonodo axilar em 76,27\% dos casos. A análise estatística revelou significância a nível de 5\%. Embora existam técnicas mais eficientes para a identificação do LS, o azul de metileno reúne as vantagens: custo mais baixo, aplicação simples e a facilidade de acesso.

Dada a importância prognóstica do comprometimento linfonodal em alguns tumores, a avaliação do LS deve ser incorporada à rotina clínica, de modo a auxiliar na determinação do prognóstico e na seleção do melhor tratamento adjuvante para as cadelas com neoplasia mamária.

\section{Agradecimentos}

Agradecemos ao CAPES pelo apoio financeiro à pesquisa e à Faculdade de Veterinária da Universidade Federal Fluminense pelo espaço cedido para execução do estudo. 


\section{Referências}

BARBOSA, E. M.; FRANCISCO, A. A. R. F.; NETO, J. T. A.; ALVES, E. M. F. A.; TAVARES, M. G. M.; GÓES, J. C. S. Fatores clínico-patológicos de predição do acometimento axilar em pacientes com metástases de câncer de mama no linfonodo sentinela. Revista Brasileira de Ginecologia e Obstetrícia, v. 32, n. 3, p. 144-149, 2010. doi: http://dx.doi.org/10.1590/S0100-72032010000300008.

BESERRA, H. E. O.; GRANDI, F.; IBAÑEZ, J. F.; ROCHA, N. S.; PINHEIRO, L. G. P. Sentinel lymph node identification: the importance of new methodologies and preclinical studies in dogs. Brazilian Journal of Veterinary Pathology, v. 6, n. 1, p. 5, 2013.

BESERRA, H. E. O.; CAVALCANTE, R. V.; PESSOA, A. W. P.; PINHEIRO, L. G. P. Técnica de detecção do Linfonodo Sentinela da glândula mamária de cadelas utilizando Azul Patente V e Tecnécio ${ }^{99 \mathrm{~m}}$ Tc. Veterinária e Zootecnia, v. 18, n. 2, p. 57-59, 2011. Suplemento 2.

CAMPOS, M. L. C.; REPETTI, C. S. F.; HATAKA, A.; MAIANTE, A. A.; SCORSATO, P. S. Pesquisa do Linfonodo Sentinela (LNS) através da administração de corante azul de metileno em cães portadores de neoplasias. Nosso Clínico, v. 10, n. 56, p. 18-34, 2007.

HEDLUND, C. S. Cirurgia do Sistema reprodutivo e genital. In: FOSSUM, T. W. Cirurgia de pequenos animais. 3. ed. São Paulo: Elsevier Inc, 2007. 1606 p.

JOHNSTON, S. D. Sistemas reprodutivos. In: SLATTER, D. Manual de cirurgia de pequenos animais. 3. ed. São Paulo: Manole, 2007.2714 p.

KHATIB, E. M.; PIRES, P. F. T. A.; LIMA, A. F. K. T.; REPETTI, C. S. F.; FRANCO, R. P.; HATAKA, A. Uso do azul de metileno na identificação do linfonodo sentinela em cadelas com neoplasias mamárias. Veterinária e Zootecnia, v. 18, n. 2 , p. 60-65, 2011. Suplemento 2.

LANA, S. E.; RUTTERMAN, G. R.; WITHROW, S. J. Tumors of the mammary gland. In: WITHROW, S. J.; VAIL, D. M. (Ed.). Small animal clinical oncology. 4. ed. St. Louis: Saunders Elsevier, 2007. p. 619-636.
NICKEL, R.; SCHUMMER, A.; SEIFERLE, E. The anatomy of domestic animals. Berlim: Verlag Paul Parey, 1981. v. 3, $630 \mathrm{p}$.

PATSIKAS, M. N.; KARAYANNOPOULOU, M.; KALDRYMIDOY, E.; PAPAZGLOU, L. G.; PAPADOPOULOU, P. L.; TZEGAS, S. I.; TZIRIS, N. E.; KAITZIS, D. G.; DIMITRIADIS, A. S.; DESSIRIS, A. K. The lymph drainage of the neoplastic mammary glands in the bitch: a lymphographic study. Anatatomy, Histology \& Embryology, v. 35, n. 4, p. 228-234, 2006. doi: http://dx.doi.org/10.1111/j.1439-0264.2005.00664.x.

PEREIRA, C. T.; MARQUES, F. L. N.; WILLIAMS, J.; MARTIN, B. W. D.; BOMBONATO, P. P. ${ }^{99 \mathrm{~m}}$ Tc-labeled dextran for mammary lymphoscintigraphy in dogs. Veterinary Radiology \& Ultrasound, v. 49, n. 5, p. 487491, 2008. doi: http://dx.doi.org/10.1111/j.17408261.2008.00414.x.

PEREIRA, C. T.; RAHAL, S. C.; CARVALHO BALIEIRO, J. C.; RIBEIRO, A. A. C. M. Lymphatic drainage on healthy and neoplasic mammary glands in female dogs: can it really be altered? Anatomia, Histologia, Embryologia, v. 32, n. 5, p. 282-290, 2003. doi: http://dx.doi.org/10.1046/j.14390264.2003.00485.x.

PINHEIRO, L. G. P.; OLIVEIRA FILHO, R. S.; VASQUES, P. H. D.; FILGUEIRA, P. H. O.; ARAGÃO, D. H. P.; BARBOSA, P. M. E.; BESERRA, H. E. O.; CAVALCANTE, R. V. Hemosiderin. A new marker for sentinel lymph node identification. Acta Cirúrgica Brasileira, v. 24, n. 6, p. 432436, 2009. doi: http://dx.doi.org/10.1590/S010286502009000600002 .

PINHEIRO, L. G. P.; MORAES, M. O. de; SOARES, A. H.; LOPES, A. J. T.; NAGUÉRE, M. A. S. P.; GONDIM, F. A. L. de; BRANDÃO, C. B.; NASCIMENTO, D. C. H.; SOARES, J. P. H.; SILVA, J. M. M. Estudo experimental de linfonodo sentinela na mama da cadela com azul patente e Tecnécio Tc99m. Acta Cirúrgica Brasileira, v. 18, n. 6, p. 514-517, 2003. doi: http://dx.doi.org/10.1590/S0102-86502003000600006. 
QUADROS, L. G. A.; GEBRIM, L. H. A pesquisa do linfonodo sentinela para o câncer de mama na prática clínica do ginecologista brasileiro. Revista Brasileira de Ginecologia e Obstetrícia, v. 29, n. 3, p. 158-164, 2007. doi: http://dx.doiorg/10.1590/S0100-72032007000300008.

RAHAL, S. C.; HOSSNE, W. S.; TEIXEIRA, E. M. S. Uso da fluorsceína na identificação dos vasos linfáticos superficiais das glândulas mamárias em cadelas. Ciência Rural, v. 25, n. 2, p. 251-254, 1995. doi: http://dx.doi.org/10.1590/S010384781995000200013 .

SAPIENZA, M. T.; TAVARES, M. G. M.; ENDO, I. S.; NETO, G. C. C.; LOPES, M. M. M. F.; NAKAGAWA, S.; BELFORT, F. A.; SOARES JÚNIOR, J.; LEWIN, S.; MARONE, M. M. S. Pesquisa do linfonodo sentinela em pacientes com melanoma: experiência com fitato marcado com Tecnécio- ${ }^{99 \mathrm{~m}}$ e revisão da literatura. Anais Brasileiros de Dermatologia, v. 79, n. 2, p. 181-191, 2004. http://dx.doi.org/10.1590/S0365-05962004000200006.

SAUTET, J. Y.; RUBERTE, J.; LOPEZ, C.; GINE, J. M.; ORDONEZZ, G.; CINGIA, A. Lymphatic system of the mammary glands in the dog: an approach to the surgical treatment of malignant mammary tumors. Canine Practice, v. 17, n. 2. p. 30-33, 1992.
SLETH, J. C. Un accident anaphylactoïde imputé au bleu patenté. Faut-il changer de colorant? Annales Françaises d'Anesthésie et de Réanimation, v. 27, n. 6, p. 515, 2008. doi: http//dx.doi.org/10.1016/jannfar.2008.05.004.

SZCZUBIAŁ, M.; ŁOPUSZYNSKI, W. Prognostic value of regional lymph node status in canine mammary carcinomas. Veterinary and Comparative Oncology, v. 9, n. 4, p. 296-303, 2011. doi: http://dx.doi.org/10.1111/j.14765829.2011.00268.x.

TUOHY, J. L.; MILGRAM, J.; WORLEY, D. R.; DERNELL, W. S. A review of sentinel lymph node evaluation and the need for its incorporation into veterinary oncology. Veterinary and Comparative Oncology, v. 7, n. 2, p. 81-91, 2009. doi: http://dx.doi.org/10.1111/j.1476-5829.2009.00183.x.

WHITE, R. A. S. Tratamento cirúrgico de distúrbios cutâneos específicos. In: SLATTER, D. (Ed.). Manual de cirurgia de pequenos animais. 3. ed. Barueri: Manole, 2007. p. 339-355.

YAMAGAMI, T.; KOBAYASHI, T.; TAKAHASHI, K.; SUGIYAMA, M. Prognosis for canine malignant mammary tumors based on TNM and histologic classification. Journal of Veterinary Medical Science, v. 58, n. 11, p. 1079-1083, 1996. doi: http://dx.doi.org/10.1292/jvms.58.11_1079. 